\title{
High-frequency Ultrasound Detection Technique with Contrast Agents
}

\author{
Jian-Xing Wu, ${ }^{1,3^{*}}$ Kuei-Hsiang Chao, ${ }^{1}$ Hsiang-Yueh Lai, ${ }^{1}$ \\ Jian-Hung Liu, ${ }^{1}$ Howard Chung, ${ }^{2}$ and Hsiao-Chuan $\mathrm{Liu}^{3}$ \\ ${ }^{1}$ Department of Electrical Engineering, National Chin-Yi University of Technology, \\ Taichung City 41170, Taiwan \\ ${ }^{2}$ Department of Internal Medicine, Queens Hospital Center, New York City, 11432, USA \\ ${ }^{3}$ Department of Radiology, Mayo Clinic, Rochester City 55905, USA
}

(Received March 20, 2019; accepted October 18, 2019)

Keywords: chirp, ultrasound, microbubble, contrast agents, imaging

High-frequency ultrasound with contrast agents provides contrast enhancement for imaging, has potential for application to drug delivery, and enables local genomics research. However, high-frequency ultrasound has a significant limitation, the achievable signal-to-noise ratio (SNR) and depth of penetration. In this study, we developed a new ultrasound system that involves chirp-coded-excitation ultrasound imaging with chirped pulses as trigger signals and a cardinal frequency of $30 \mathrm{MHz}$. A chirp is a coded signal that linearly spans a frequency bandwidth $B$ $=f_{2}-f_{1}$, where $f_{1}$ and $f_{2}$ are the starting and ending frequencies, respectively. A new chirped pulses with contrast agents to minimize the attenuation of energy in human tissues increased the signal-to-noise ratio (SNR) by $20 \mathrm{~dB}$ for high-frequency ultrasonic flow imaging of the heart of zebrafish and increased the penetration depth to $2.2 \mathrm{~mm}$ with pulse compression and a handmade expander. On the other hand, in a microbubble experiment, adopting various echo signal concentrations resulted in the desired distribution of different types of microbubbles. Using the system we developed, we experimentally demonstrated that the chirp-coded excitation reduces the SNR by about $43 \mathrm{~dB}$ compared with unipolar and bipolar pulse excitations.

\section{Introduction}

Recently, chirp-coded excitation has been found to have the potential to enhance imaging locally and hence improve local drug delivery. The attenuation of ultrasound is proportional to its frequency in the human body. The higher the ultrasound frequency, the severer the ultrasound attenuation through tissue. Generally, owing to this attenuation effect, which decreases the ultrasound energy, a high-frequency ultrasound signal is weaker than a lowfrequency ultrasound signal. Moreover, a high-frequency ultrasound signal is more easily affected by the noise of internal and external systems than a low-frequency ultrasound signal, limiting the signal-to-noise ratio (SNR) and sensitivity. By keeping the system noise constant, we can improve the SNR of a high-frequency ultrasound imaging system. ${ }^{(1,2)}$ There are two methods of increasing the trigger signal energy. The first method is to increase the amplitude of "Corresponding author: e-mail: jian0218@gmail.com https://doi.org/10.18494/SAM.2019.2382 
the trigger signal for a higher system sensitivity. Typically, the pulse amplitude of commercial ultrasound systems has already been raised to the maximum mechanical index (MI). The second method is to lengthen the duration of the transmitted signal to increase the average power of the trigger signal. This processed signal is called a coded waveform. The use of the coded waveform is proposed for increasing the SNR and penetration depth of the ultrasound system. However, the pulse duration might also be increased. It is noteworthy that the length of the transmitted signal determines the axial resolution. The chirp-coded excitation technique is utilized to recover the axial resolution of images by transforming the average power of the coded waveform to the instantaneous power of a short pulse, thus increasing the signal amplitude. In this study, we used the chirp signal excitation technique to raise the signal energy so that the echo signal can achieve a higher image resolution. After that, pulse compression is applied to the echo signal to recover the axial resolution. We use phantoms and wire phantoms to compare the characteristics of the coded waveform with those of a short pulse. Then, the coded excitation is employed in contrast imaging to enhance the image quality. ${ }^{(3,4)}$

The chirp-coded excitation technique affects the SNR and axial resolution of an ultrasound image. A short pulse results in worse penetration depth and SNR. Nonetheless, the bandwidth of a short pulse is large, which corresponds to a high axial resolution. On the other hand, a long pulse, which has a higher energy, exhibits better penetration depth and SNR, but worse axial resolution owing to its narrow band. Coded signals are one type of long pulse. Coded excitation extends the bounds of this tradeoff by increasing the SNR and penetration depth through appropriate coding on the transmitting part and decoding on the receiving part. In singletransmit codes, a single sequence must be compressed by a compression filter. In contrast, in multiple-transmit systems, two or more courses must be combined to achieve compression. One of the most common phase-modulated codes is the biphase, and the representative waveforms are Barker and Golay codes with symbols \pm 1 .

The most common frequency modulation is the swept-frequency chirp. The instantaneous frequency of the chirp is known to change linearly with time. ${ }^{(5)}$ In the same bandwidth, the pulse of this chirp is longer than those of other chirps such as the Gaussian chirp. A longer pulse has a higher energy. Thus, it is difficult to attenuate such a pulse, and the SNR is increased in the end. However, a longer pulse also causes the overlapping of echo signals, which further affects the axial resolution. Under such circumstances, a compression filter is used to squeeze a long pulse into a short pulse to obtain a higher axial resolution in general. The following are the characteristics of the desired chirp: (1) long pulse duration; (2) the instantaneous frequency of the chirp increases with time (including the components from low to high frequencies). ${ }^{(6)}$ We chose the chirp whose frequency changes with time to be the excitation signal. It can match the frequency response of sensors accurately. The trigger signal can be efficiently transmitted, and the nonlinear phase can be eliminated by the following pulse compression. ${ }^{(7-9)}$ Figure 1(a) shows the output of the arbitrary waveform generator (AWG). As shown in Fig. 1(b), the power amplifier amplifies the chirp signals above $100 \mathrm{~V}$ as trigger signals to the trigger sensor.

In the proposed system, we develop an ultrasound system that uses the chirp waveform as the trigger signal to improve the SNR and penetration depth by increasing the average power of 


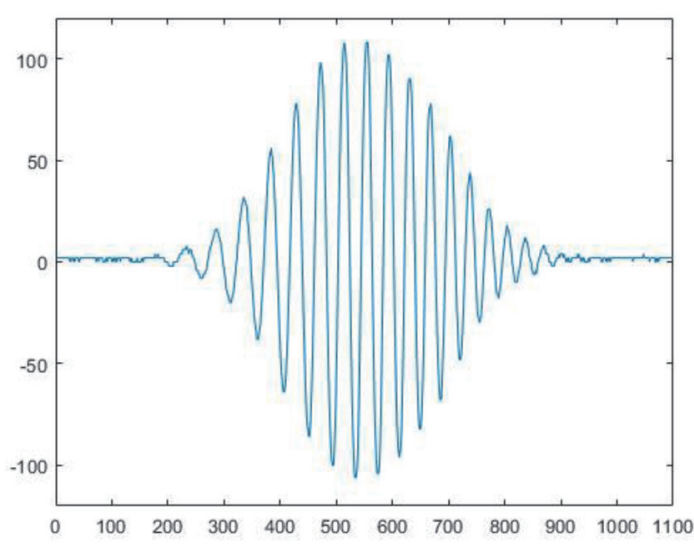

(a)

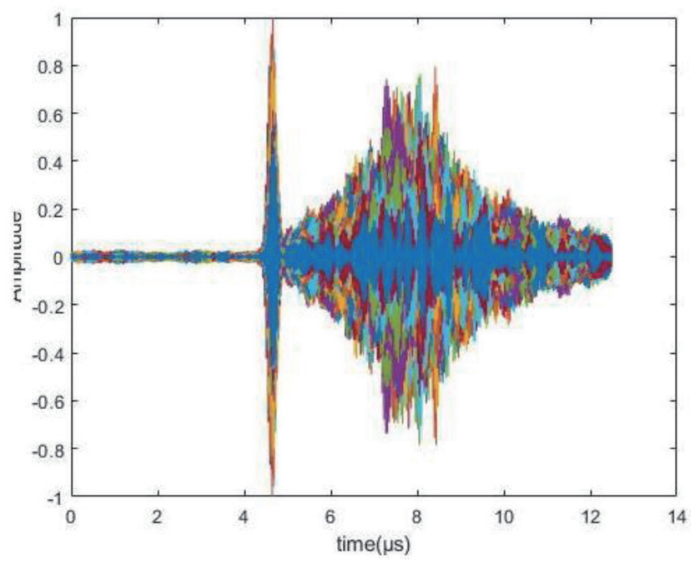

(b)

Fig. 1. (Color online) (a) Simulated single RF-line data of chirp. (b) Chirp after amplification with no attenuation.

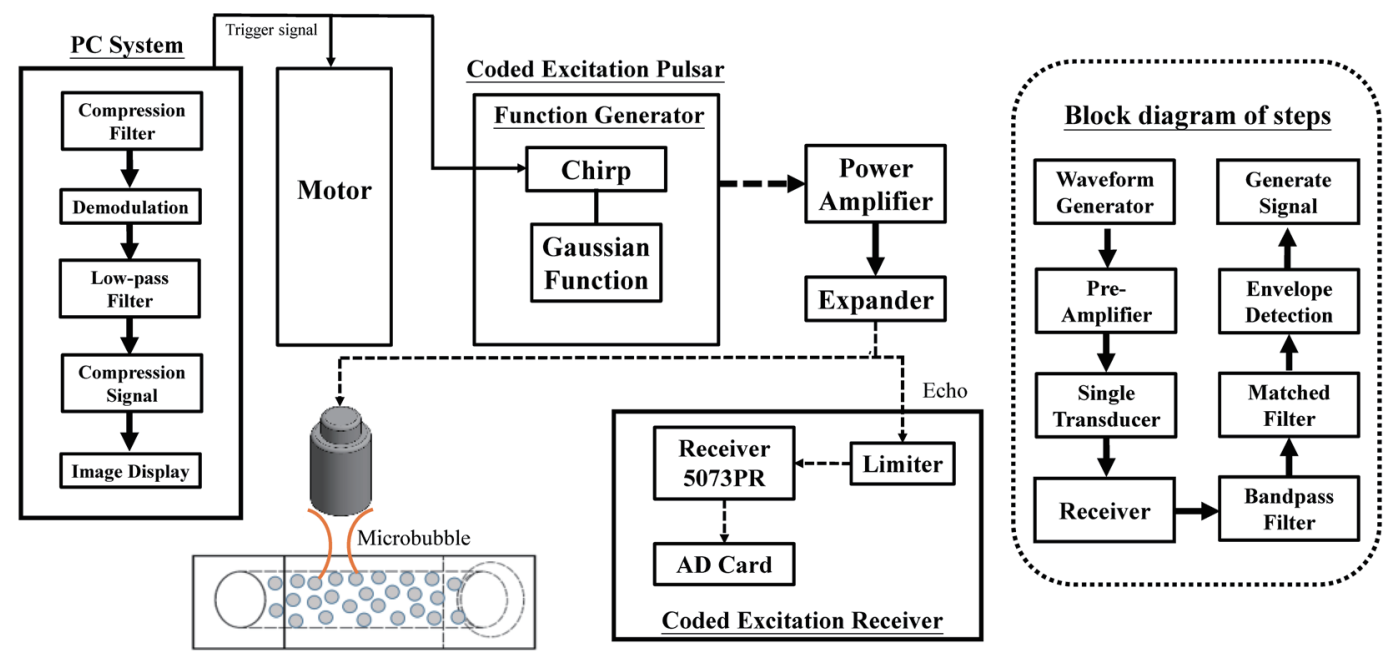

Fig. 2. (Color online) Block diagram of chirp-coded-excitation ultrasound system.

the signal. Figure 2 shows the block diagram of the chirp-coded-excitation ultrasound system, which is divided into two parts: the hardware of the ultrasound system, including the arbitrary waveform generator, and the arbitrary waveform power amplifier.

\section{Materials and Methods}

\subsection{Coded excitation signal}

In an ultrasound imaging system, to improve the system effectiveness, in commercial ultrasound pulse systems (5900, PANAMETRICS 5900PR, OLYMPUS), the trigger signal is increased to its maximum MI. For safety concerns, the transmitted signal energy is limited 
to prevent tissue damage caused by the high instantaneous pressure of ultrasound, such as overheating of tissue and cavitation effects. Ordinary short-pulse energy is defined by the power amplitude, rather than by the average power, of the trigger signal. ${ }^{(10-14)}$ Therefore, the chirp-coded excitation can increase the average energy of the signal without affecting the amplitude of the trigger signal to yield better SNR and penetration depth. ${ }^{(15)}$

The bandwidth envelope of a long pulse is narrower than that of a short pulse in the spectrum. Hence, a long-pulse echo could not be compressed by the compression filter to recover the axial resolution. In this research, we used a chirp as a carrier to make the bandwidth of a long pulse similar to that of the short pulse. Chirp frequency increases with time so that the bandwidth of a chirp is more significant than that of a single-frequency sine wave carrier. Using the chirp as a carrier of a long pulse can increase the bandwidth and also help in echo compression. The chirp is described as

$$
c(t)=w(t) \cos \left[2 \pi\left(f_{0}-\frac{\Delta f}{2} t+\alpha t^{2}\right)\right], 0 \leq t \leq T,
$$

where $w(t)$ is the Gaussian window, $f_{0}$ is the carrier frequency, $\Delta f$ is the chirp bandwidth, $T$ is the signal duration, and $\alpha$ is the rate of frequency increase $(\alpha=2 \Delta f / T)$. The chirp signal duration is adjusted by the compression ratio function. The Gaussian envelope multiplied by the chirp signal is the Gaussian chirp pulse. The Gaussian chirp pulse is adjusted by changing the chirp duration to obtain different signal durations with constant bandwidths.

\subsection{System descriptions}

In this study, we used an ultrasound transducer with a center frequency of $30 \mathrm{MHz}$ (SEUT306, Acoustic Sensor Co., Ltd.) and an element diameter of $6 \mathrm{~mm}$. According to Fig. 3, the bandwidth of the sensor is higher than $82 \%$ and the focal depth is $12 \mathrm{~mm}$. Moreover, there

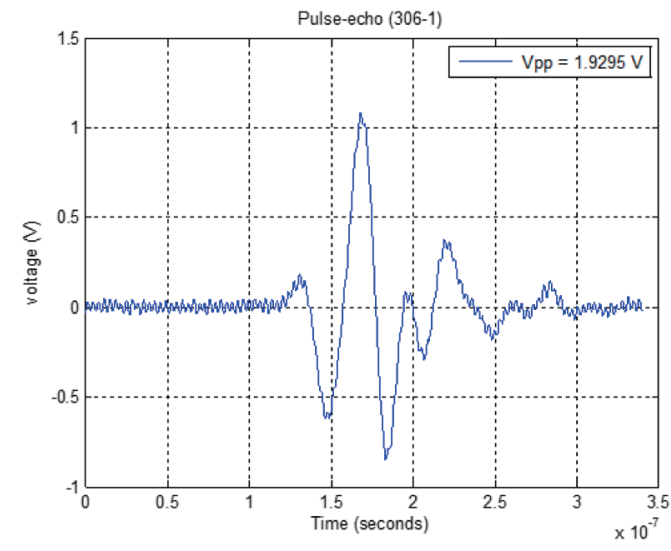

(a)

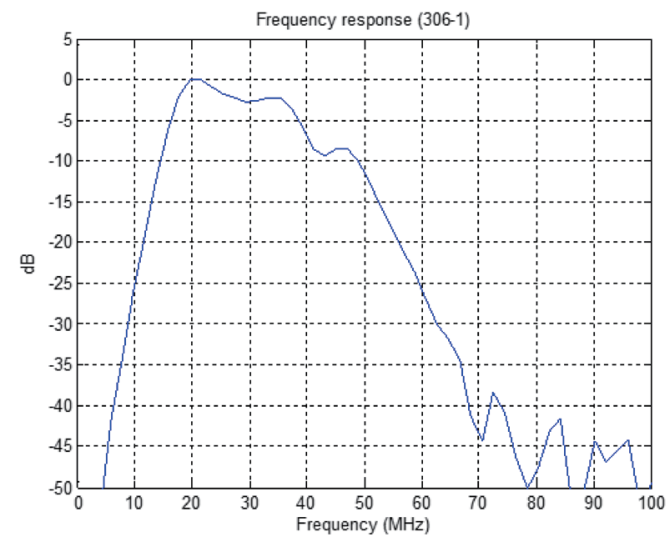

(b)

Fig. 3. (Color online) (a) Pulse echo waveform and (b) frequency response. 
is a protective parylene-c layer, which acts as a matching layer, coating the transducer and brass housing. This layer is used to increase the transmission efficiency and the ultrasound transmittance back to the sensor. ${ }^{(16-19)}$

The pulse system included a $240 \mathrm{MHZ}$ sample/s function generator (AFG3252, Tektronix Inc.) and an arbitrary waveform power amplifier (model 325LA, Electronics \& Innovation Ltd., USA). Computer programs can generate arbitrary waveforms that are transferred by the spatial software and input to the arbitrary waveform generator. Therefore, the arbitrary waveform generator can generate various transmission signals. The nominal gain of $325 \mathrm{LA}$ is $50 \mathrm{~dB}$ and the variation of gain is within $\pm 1.5 \mathrm{~dB}$. Moreover, the input voltages of 325LA have a flat frequency response from $250 \mathrm{kHz}$ to $125 \mathrm{MHz}$. Input voltages exceeding twice the voltage peak may permanently damage the instrument. In this study, we use 5073PR as an ultrasound receiver and the maximum bandwidth ranges from $1 \mathrm{kHz}$ to $75 \mathrm{MHz}$. The noise of the signal affecting the receiver is $7 \mu \mathrm{V}$ peak-to-peak. After MATLAB edits the chirp waveform as the trigger signal input to the function generator, the power amplifier amplifies the trigger signal to generate a high-voltage pulse. ${ }^{(20-24)}$ The trigger signal goes through the diode circuit (expander) to filter noise, because the noise is amplified by the power amplifier to affect the trigger signal, and then the high-frequency transducer is triggered by the trigger signal to transmit the ultrasound signal. ${ }^{(25-27)}$ Before reaching the receiver, the echo signal goes through the other diode circuit (limiter) to prevent the high-voltage trigger signal from penetrating the receiving part to protect the instruments. Next, the receiver, 5073PR, receives and appropriately amplifies the echo signal. Then, the echo signal is transferred to images with signal processing. In the fixed position part, using a three-dimensional motion system, a transducer for scanning images is attached. The primary scan control motor is a ceramic linear servo motor (HR8) that controls the direction of the $x$-axis. The motor of the $y$ - and $z$-axes is a high-precision stepper motor that controls the motion. The use of the three-dimensional motion system enables the position to be spatially fixed in three dimensions. Table 1 shows the specifications of the three-dimensional motion system.

\subsection{Signal processing}

Ultrasound signal processing and imaging are separated into two parts. The first involves compression filtering to eliminate the phase difference of the echo signal and focus the energy of the echo signal to recover the axial resolution. A matched filter is chosen as a compression filter in this study. The matched filter is placed on the ideal ultrasound signal. Therefore, the echo signal energy is focused to achieve pulse compression. The second part is demodulation.

Table 1

Specifications of three-dimensional motion system.

\begin{tabular}{lcccc}
\hline Axis & Stroke $(\mathrm{mm})$ & Count $(\mu \mathrm{m})$ & Load $(\mathrm{kg})$ & Velocity $(\mathrm{mm} / \mathrm{s})$ \\
\hline$x$ & 150 & 0.1 & 2 & 150 \\
$y$ & 300 & 0.8 & 10 & 100 \\
$z$ & 50 & 0.6 & 7 & 50 \\
\hline
\end{tabular}


The echo signal is demodulated to diversify the wave crest and filter the carrier with the ultrasound transducer. Then, the compressed signal will be transformed into B-mode images. When the ultrasound system scans images, the trigger signal is generated by an arbitrary waveform generator. The trigger signal then passes through an arbitrary waveform amplifier to be amplified and triggers the transducer to produce an ultrasound signal. The echo signal is received and appropriately amplified by the receiver and then input to the oscilloscope for display. A test block diagram of the experimental system is demonstrated. In the system test, the oscilloscope shows that the echo signal covered with noise is difficult to observe. Therefore, the output signal of every instrument is examined to locate and reduce the noise source. An appropriate receiver is chosen to enhance the SNR.

In a conventional radar and ultrasound system, to simultaneously achieve better system SNR and spatial resolution, Schottky diodes are applied to eliminate nonlinear noise. Ordinary Schottky diodes, 1N4148 and 1N914, are manufactured into expanders. Three types of expanders are connected with the power amplifier, and then the echo signal is set to $0.5 \mathrm{~V}$ chirp with a trigger signal $(30 \mathrm{MHz})$ to measure its SNR. Table 2 shows the measured results. Both commercial and handmade expanders can reduce the noise of the power amplifier. The sound is decreased to $0.04 \mathrm{~V}$, so the SNR of the echo signal is efficiently improved. For the echo signal, the SNR of expander 1N4148 is better than those of the others, so IN4148 will be used in this study to reduce the noise.

\subsection{Microbubble imaging}

When microbubbles are used, a discussion on their concentration is vital. If the microbubble concentration is too low, the scatter signal will be too weak to enhance the contrast of images. In contrast, if the microbubble concentration is too high, the ultrasound signal will be severely attenuated and the back part of the object cannot be imaged. This is called the shielding effect. Therefore, an appropriate microbubble concentration must be found. Considering also that microbubbles will be used for in vitro experiments, the determination of the appropriate microbubble concentration is essential. When microbubbles are injected into small animals, they will be diluted by their blood circulation, reducing the intensity of the scatter signal. However, if the concentration of microbubbles injected into small animals is too high, thrombosis will occur. Therefore, by choosing an appropriate microbubble concentration that can be diluted by the blood circulation of an animal, the microbubbles will retain their strong scattering ability and will not cause thrombosis. The microbubbles with $\mathrm{C}_{3} \mathrm{~F}_{8} 1.1 \mu \mathrm{m}, \mathrm{C}_{3} \mathrm{~F}_{8}$ $2 \mu \mathrm{m}, \mathrm{C}_{4} \mathrm{~F}_{10} 1.1 \mu \mathrm{m}$, and $\mathrm{C}_{4} \mathrm{~F}_{10} 2 \mu \mathrm{m}$ are used in this study. Assume that the microbubbles injected into a small animal are diluted by the blood circulation with time. The microbubble

Table 2

Results of the echo signal measured with different expanders.

\begin{tabular}{lccc}
\hline & Commercial expander & Expander 1N414B & Handmade expander \\
\hline Peak voltage & $1.38 \mathrm{~V}$ & $1.42 \mathrm{~V}$ & $1.38 \mathrm{~V}$ \\
Noise voltage & $0.04 \mathrm{~V}$ & $0.04 \mathrm{~V}$ & $0.04 \mathrm{~V}$ \\
SNR & $30.75 \mathrm{~dB}$ & $31 \mathrm{~dB}$ & $30.75 \mathrm{~dB}$ \\
\hline
\end{tabular}


concentration decreases in the range of $10-100 \%$ in steps of $10 \%$. The diluted microbubbles are injected into an acrylic phantom and then into the ultrasound scanner. Terason model t3000 (Teratech Corporation, USA) is used to scan the images. On the basis of the brightness of images with different microbubbles concentrations, the appropriate concentration ranges for four types of microbubbles are found. Ultrasound contrast agents are injected into the blood circulation by intravenous injection to enhance the signal of the blood zone to detect microperfusion. The characteristic of microbubble destruction and the size distribution of microbubbles after dissolution are essential information in drug release.

When the signal frequency is closer to the resonant frequency, the image contrast is higher. However, the trigger function in the chirp-coded-excitation ultrasound system has a high frequency, so the microbubble size must be less than $1 \mu \mathrm{m}$, which is not very common. Consequently, large microbubbles are destroyed while small microbubbles remain to enhance the image contrast. The parameters of the destruction signal are three acoustic pressures $(0.2,0.4$, and $0.6 \mathrm{MPa}$ ) and three signal cycles (100, 500, and 1000 cycles). Microbubbles do not remain to have the same size in the ultrasound scan. Microbubbles expand or contract with positive- or negative-voltage transformation, respectively. Therefore, different acoustic pressures are set to observe the size distribution of microbubbles after their destruction. Signal cycles will affect the microbubble oscillation time. When the signal cycles reach a threshold, a microbubble fragments into many smaller microbubbles. The number of microbubbles will then increase, but their size will decrease. Hence, different numbers of signal cycles are set to observe the changes in the number of microbubbles. The microbubbles are injected into an acrylic phantom. The function generator is used to generate a destruction signal with the above-mentioned parameters. The signal passes through the power amplifier to be amplified and then is input to a $1 \mathrm{MHz}$ transducer (model V303, Panametrics, Waltham, MA) to produce the signal that destroys microbubbles. The pulse repetition frequency (PRF) is $1 \mathrm{~Hz}$ and the destruction time is $30 \mathrm{~s}$.

\section{Experimental Results and Discussion}

\subsection{Image phantom manufacture}

In ultrasound image research, the wire phantom can be a useful reference phantom in the analyses of the axial and lateral resolutions, system noise, and dynamic range in an ultrasound system. A wire phantom consisting of five 20- $\mu \mathrm{m}$-diameter tungsten wires (Goodfellow Cambridge Ltd., England) is used to evaluate the image resolution. The cables are diagonally aligned, with axial and lateral spacings of 1 and $2 \mathrm{~mm}$, respectively. Therefore, unipolar pulse, bipolar pulse, and chirp-coded excitations are used to scan images to compare their efficacies. Figure 4 shows the images of wire phantoms with different excitations. Figure 5 shows the magnitude of the echo signal from the wire around the focal point for short vibration and chirp-coded excitation. The SNR for short pulse is $31 \mathrm{~dB}$ and that for coded excitation is $43 \mathrm{~dB}$. Hence, the SNR of the proposed system increased to $12 \mathrm{~dB}$ compared with that of the commercial ultrasound pulse system in the case of chirp-coded excitation. 


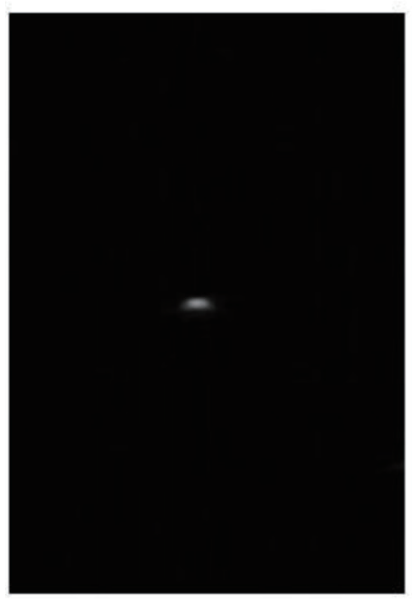

(a)

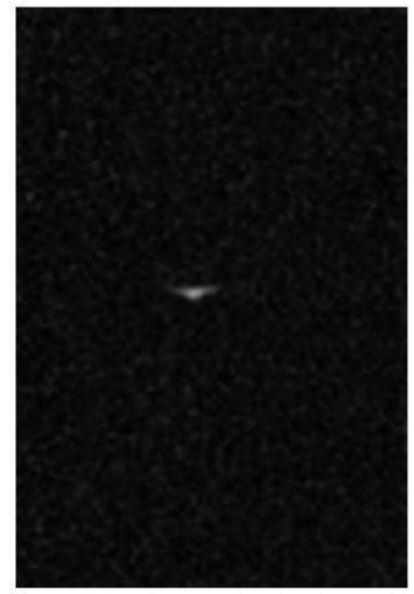

(b)

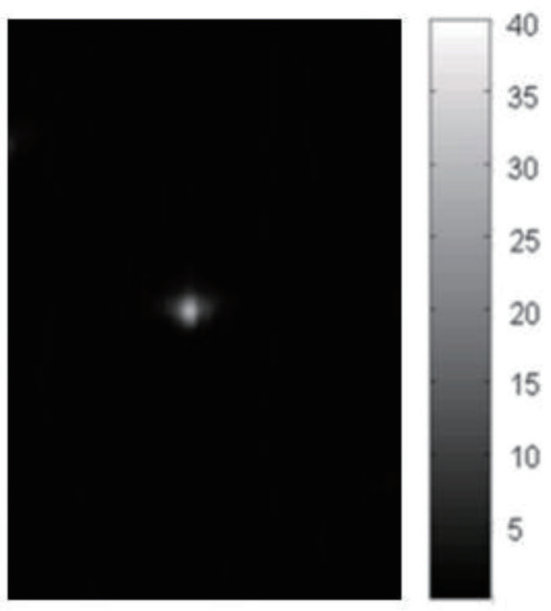

(c)

Fig. 4. Images of wire phantom. (a) Unipolar pulse imaging. (b) Bipolar pulse imaging. (c) Chirp-coded-excitation imaging.

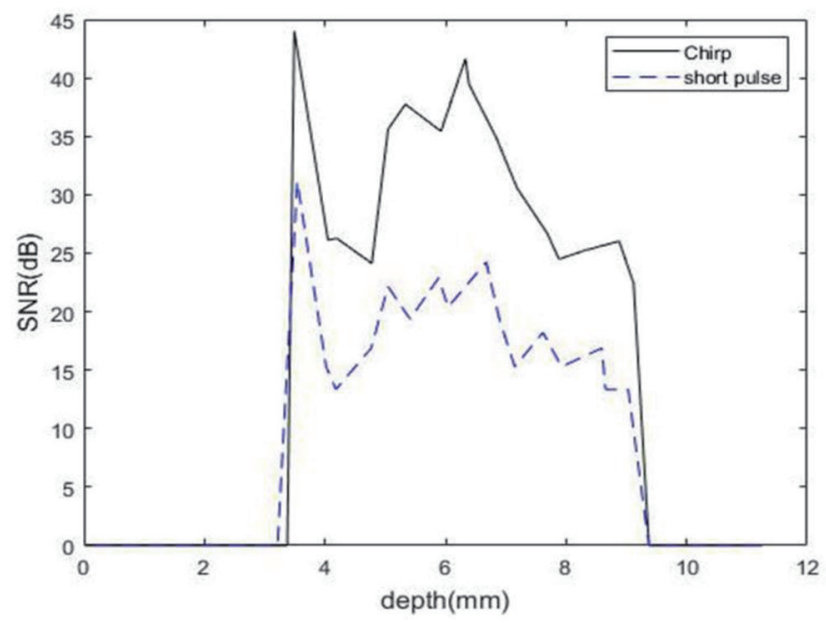

Fig. 5. (Color online) SNR for short pulse and chirp-coded excitation as a function of depth.

In the images of the wire phantom, the wire should appear circular, but the wire images are distorted because the cable is out of the focal point. Therefore, the ratio of the minor axis to the major axis of the deformed wire images is a parameter that represents the amount of deformation in a different position as a function of depth. The larger the amount of deformation, the farther the distance between the wire and the focal point. The deformation in the case of a short pulse is severer than that in the case of coded excitation. Therefore, a soft tissue phantom ( $8 \mathrm{~cm}$ long, $5 \mathrm{~cm}$ wide, $1.5 \mathrm{~cm}$ high) was used to simulate the real conditions under which the ultrasound would be applied, and the results were compared with an ultrasound image for a 
different type of excitation. A chirp-coded excitation and a short pulse with a trigger signal (30 $\mathrm{MHz}$ ) were used to scan the tissue phantom. Figure 6 shows the images of the tissue phantom obtained with different types of coded excitation. The coded excitation, compared with the unipolar pulse excitation, increased the penetration depth to $2 \mathrm{~mm}$.

\subsection{Microbubble imaging}

There are four types of microbubbles used in this study. Finding the appropriate concentration range prevents the microbubble concentration from being too low to enhance the image contrast or too high that it causes the ultrasound signal to attenuate severely. Therefore, the four types of microbubbles are diluted into ten concentrations. Figure 7 shows contrast images of four types of microbubbles at four different levels. The trigger signal in the chirpcoded-excitation ultrasound system is a high-frequency signal. When the signal frequency is closer to the resonant frequency of microbubbles, the image contrast is much higher. However, the size of the microbubbles is inversely proportional to the resonant frequency. The smaller the microbubbles, the higher the resonant frequency. In contrast, the larger the microbubbles, the lower the resonant frequency.

Recently, the heart of the zebrafish has become a powerful model for understanding selfhealing ability, so the zebrafish plays a vital role in regenerative medicine. Therefore, to observe zebrafish, microbubbles are used to enhance the image contrast. Figure 8 shows the difference in the zebrafish heart before and after microbubble injection. Figure 8(a) shows the heart of the zebrafish without microbubbles: only the contour of the breast can be observed. Figure 8 (b) shows the heart of the zebrafish after microbubble injection. The ventricle and the atrium of the heart can be seen.

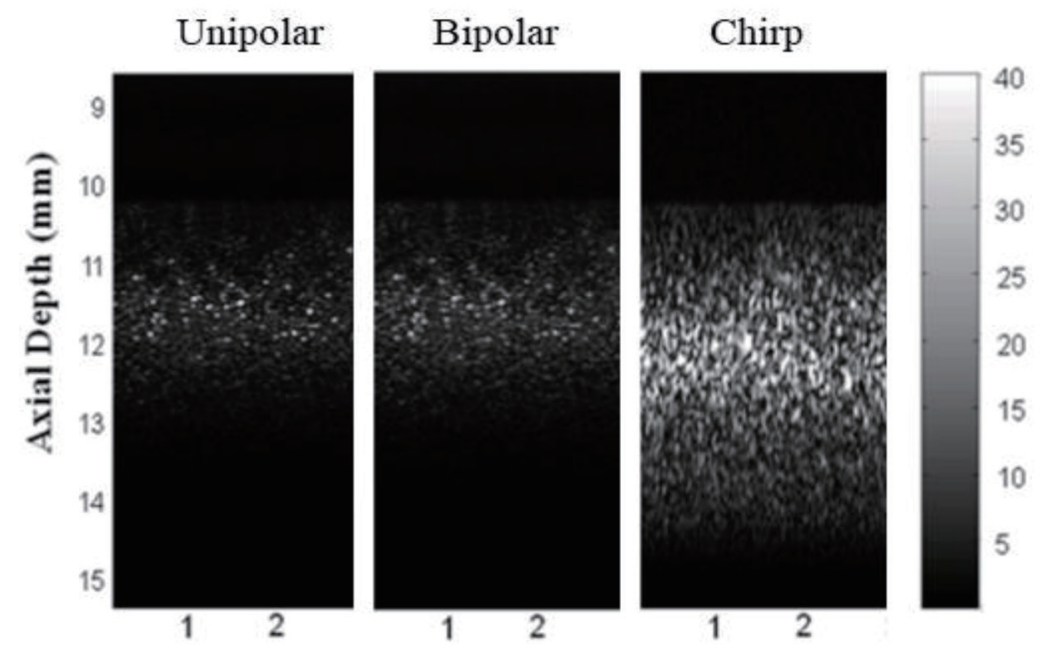

\section{Lateral Distance (mm)}

Fig. 6. Images of ultrasound phantom. (a) Unipolar pulse excitation. (b) Bipolar pulse excitation. (c) Chirp-coded excitation. 


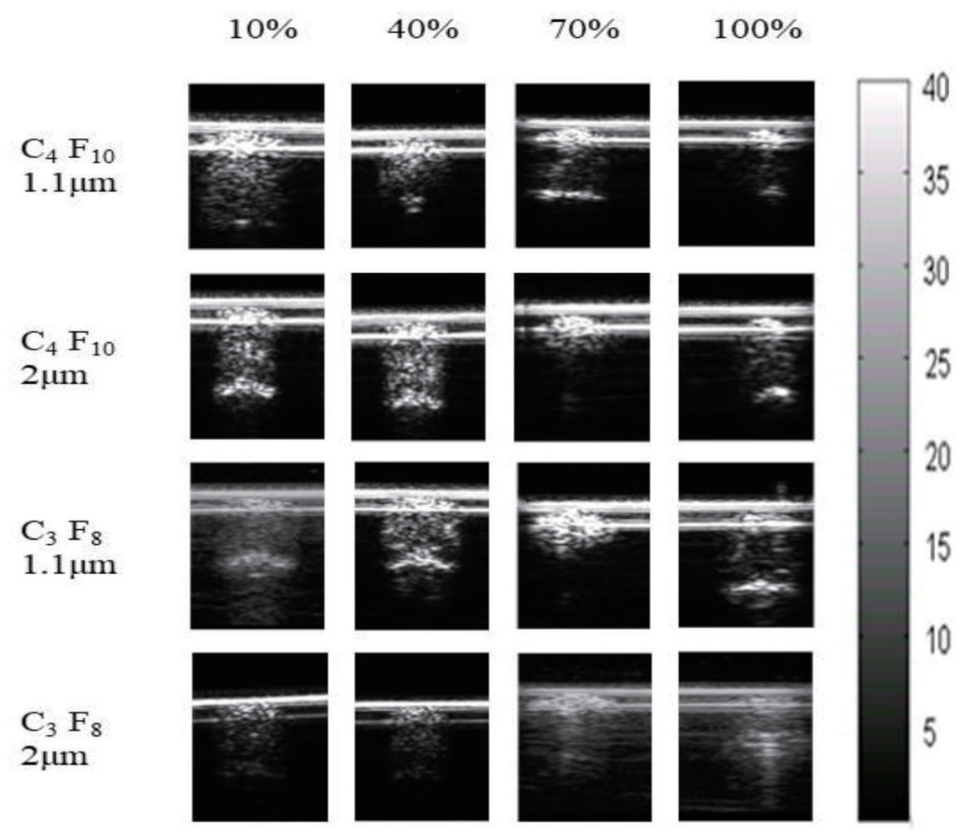

Fig. 7. Contrast images for four microbubble concentrations: $\mathrm{C}_{3} \mathrm{~F}_{8} 1.1 \mu \mathrm{m}, \mathrm{C}_{3} \mathrm{~F}_{8} 2 \mu \mathrm{m}, \mathrm{C}_{4} \mathrm{~F}_{10} 1.1 \mu \mathrm{m}$, and $\mathrm{C}_{4} \mathrm{~F}_{10}$ $2 \mu \mathrm{m}$.
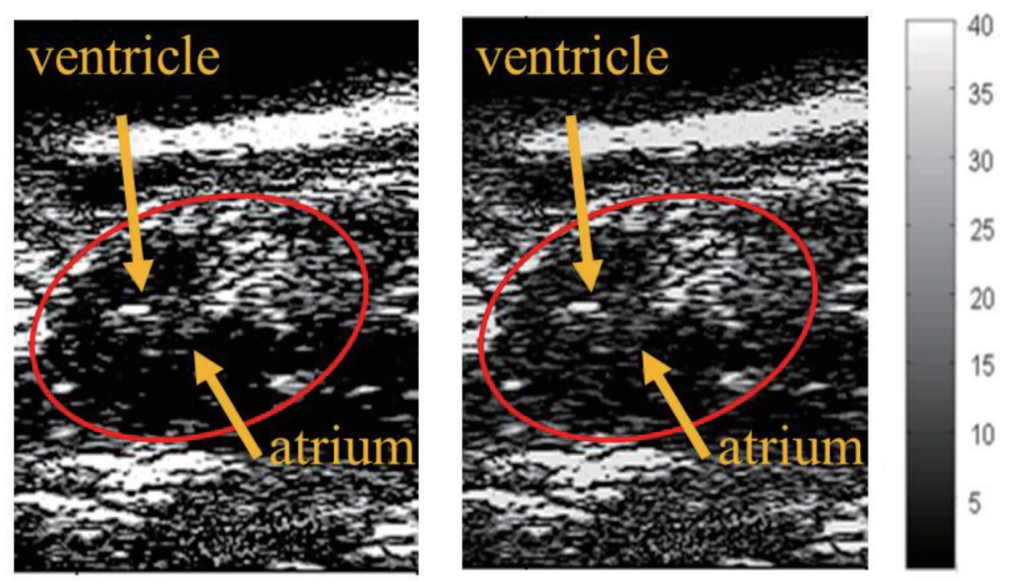

Fig. 8. (Color online) (a) Heart of zebrafish without microbubbles. (b) Heart of zebrafish after microbubble injection.

\section{Conclusions}

In this study, we evaluated the performance of the high-frequency ultrasound detection technique with contrast agents. As a result, we verified both qualitatively and quantitatively that chirp-coded excitation using an appropriate receiver and handmade expander leads to an SNR improvement of about $12 \mathrm{~dB}$ compared with short-pulse excitation. However, the microbubble 
imaging experiment yielded the following appropriate concentration ranges for four types of microbubbles injected in vivo: $\mathrm{C}_{3} \mathrm{~F}_{8}, 1.1 \mu \mathrm{m}$ from 20 to $40 \% ; \mathrm{C}_{3} \mathrm{~F}_{8}, 2 \mu \mathrm{m}$ from 80 to $100 \%$; $\mathrm{C}_{4} \mathrm{~F}_{10}, 1.1 \mu \mathrm{m}$ from 20 to $40 \%$; and $\mathrm{C}_{4} \mathrm{~F}_{10}, 2 \mu \mathrm{m}$ from 30 to $50 \%$. In the specific setting of the destruction signal, the microbubble size distribution decreased. Therefore, when the signal frequency was closer to the resonant frequency of the microbubbles, the image contrast was enhanced. The microbubbles were injected into a zebrafish, and then the zebrafish was scanned with the chirp-coded-excitation ultrasound system to observe the changes in the heart before and after microbubble injection. In the future, the chirp-coded-excitation ultrasound system with microbubbles may be used to scan tumors in a small animal without dissection. They may also be used as carriers of medicines, which are burst to release them at a specific area, because a chirp has a higher energy and hence can achieve better penetration and SNR.

\section{Acknowledgments}

This work was supported by the Ministry of Science and Technology, Taiwan, contract number MOST 108-2218-E-167-007-MY2. The authors are thankful for the technical assistance of Professor Chih-Kuang Yeh of National Tsing Hua University and for helpful discussion on the animal model.

The authors' contributions are as follows: conceptualization, Kuei-Hsiang Chao and HsiangYueh Lai; methodology, Jian-Xing Wu and Jian-Hung Liu; validation, Howard Chung and Hsiao-Chuan Liu; resources, P. Israsena; writing_-original draft preparation, Jian-Xing Wu and Hsiao-Chuan Liu; supervision, Jian-Xing Wu and Hsiao-Chuan Liu.

\section{References}

1 J. Kang, Y. Kim, W. Lee, and Y. Yoo: IEEE Trans. Ultrason. Ferroelectr. Freq. Control 64 (2017) 1698. https:// doi.org/10.1109/TUFFC.2017.2748165

2 J. Park, C. Hu, and K. K. Shung: IEEE Trans. Ultrason. Ferroelectr. Freq. Control 58 (2011) 2620. https://doi. org/10.1109/TUFFC.2011.2125

3 H. Shekhar, N. J. Smith, J. L. Raymond, and C. K. Holland: Ultrasound Med. Biol. 44 (2017) 434. https://doi. org/10.1016/j.ultrasmedbio.2017.09.021

4 I. Beekers, T. van Rooij, M. D. Verweij, M. Versluis, N. de Jong, S. J. Trietsch, and K. Kooiman: IEEE Trans. Ultrason. Ferroelectr. Freq. Control 65 (2018) 570. https://doi.org/10.1109/TUFFC.2018.2803137

5 W. Qiu, Y. Yu, F. K. Tsang, H. Zheng, and L. Sun: IEEE Trans. Biomed. Eng. 60 (2013) 1184. https://doi. org/10.1109/TBME.2013.2244887

6 J.-X. Wu, Y.-C. Du, C.-H. Lin, P.-J. Chen, and T. Chen: 2013 IEEE Int. Ultrason. Symp. (IEEE, 2013) 15711574. https://doi.org/10.1109/ULTSYM.2013.0400

7 J.-H. Liu, G.-S. Jeng, T.-K. Wu, and P.-C. Li: IEEE Trans. Ultrason. Ferroelectr. Freq. Control 53 (2006) 1590. https://doi.org/10.1109/TUFFC.2006.1678187

8 J.-X. Wu, C.-H. Lin, Y.-C. Du, P.-J. Chen, C.-C. Shih, and T. Chen: IEEE Int. Ultrason. Symp. (IEEE, 2015) 1-4. https://doi.org/10.1109/ULTSYM.2015.0328

9 I. Beekers, T. van Rooij, A. F. W. van der Steen, N. de Jong, M. D. Verweij, and K. Kooiman: IEEE Trans. Ultrason. Ferroelectr. 66 (2019) 244. https://doi.org/10.1109/TUFFC.2018.2881724

10 J. Li, Y. Wang, B. Lei, J.-Z. Cheng, J. Qin, T. Wang, S. Li, and D. Ni: IEEE J. Biomed. Health. Inf. 22 (2018) 215. https://doi.org/10.1109/JBHI.2017.2703890

11 M. Denis, M. Mehrmohammadi, P. Song, D. D. Meixner, R. T. Fazzio, S. Pruthi, D. H. Whaley, S. Chen, M. Fatemi, and A. Alizad: PloS One 10 (2015) e0119398. https://doi.org/10.1371/journal.pone.0119398

12 I. Beekers, T. van Rooij, M. D. Verweij, M. Versluis, N. de Jong, S. J. Trietsch, and K. Kooiman: IEEE Trans. Ultrason. Ferroelectr. Freq. Control 65 (2018) 570. https://doi.org/10.1109/TUFFC.2018.2803137 
13 B. H. A. Lammertink, R. Deckers, M. Derieppe, I. De Cock, I. Lentacker, G. Storm, C. T. W. Moonen, and C. Bos: Mol. Imag. Biol. 19 (2017) 683. https://doi.org/10.1007/s11307-016-1042-x

14 J. Sijl, M. Overvelde, B. Dollet, V. Garbin, N. de Jong, D. Lohse, and M. Versluis: J. Acoust. Soc. Amer. 129 (2011) 1729. https://doi.org/10.1121/1.3505116

15 K. Kooiman, H. J. Vos, M. Versluis, and N. de Jong: Adv. Drug Delivery Rev. 72 (2014) 28. https://doi. org/10.1016/j.addr.2014.03.003

16 I. Lentacker, I. De Cock, R. Deckers, S. C. De Smedt, and C. T. W. Moonen: Adv. Drug Delivery Rev. 72 (2014) 49. https://doi.org/10.1016/j.addr.2013.11.008

17 H. Shekhar, R. T. Kleven, T. Peng, A. Palaniappan, K. B. Karani, S. Huang, D. D. McPherson, C. K. Holland: Sci. Rep. 9 (2019) 9902. https://doi.org/10.1038/s41598-019-46112-z

18 T. Van Rooij, Y. Luan, G. Renaud, A. F. W. van der Steen, M. Versluis, N. de Jong, and K. Kooiman: Ultrasound Med. Biol. 41 (2015) 1432. https://doi.org/10.1016/j.ultrasmedbio.2015.01.004

19 J.-X. Wu, P.-T. Huang, C.-H. Lin, and C.-M. Li: IET Healthcare Techno. Lett. 5 (2018) 38. https://doi. org/10.1049/htl.2017.0091

20 J. E. Chomas, P. Dayton, D. May, and K. Ferrara: J. Biomed. Opt. 6 (2001) 141. https://doi.org/10.1117/1.1352752

21 L. Sun, W. D. Richard, J. M. Cannata, C. C. Feng, J. A. Johnson, J. T. Yen, and K. K. Shung: IEEE Trans. Ultrason. Ferroelectr. Freq. Control 54 (2007) 1648. https://doi.org/10.1109/TUFFC.2007.436

22 N. De Jong: Eng. Med. Biol. Mag. 15 (1996) 72. https://doi.org/10.4103/2303-9027.193594

23 P. J. A. Frinking, A. Bouakaz, J. Kirkhorn, F. J. T. Cate, and N. De Jong: Ultrasound Med. Biol. 26 (2000) 965. https://doi.org/10.1016/S0301-5629(00)00229-5

24 J. R. Lindner: Nat. Rev. Drug Discovery 3 (2004) 527. https://doi.org/10.1038/nrd1417

25 J.-X. Wu, Y.-C. Du, M.-J. Wu, C.-M. Li, C.-H. Lin, and T. Chen: Sci. World J. 2014 (2014) 1. https://doi. org $/ 10.1155 / 2014 / 203148$

26 P.-C. Li: Ultrasonic Imaging 21 (1999) 1. https://doi.org/10.1177/016173469902100101

27 C.-K. Yeh, S.-Y. Su, and W.-S. Chen: J. Med. Biol. Eng. 25 (2005) 159. https://doi.org/10.1117/1.1352752

\section{About the Authors}

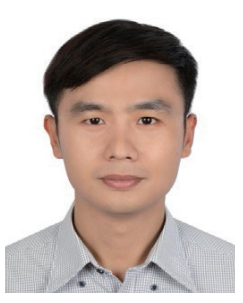

Jian-Xing Wu was born in 1985. He earned his Ph.D. degree from the Department of Biomedical Engineering at National Cheng Kung University in 2014. From 2014 to 2017, he was a Postdoctoral Research Fellow at National Synchrotron Radiation Research Center, Hsinchu, Taiwan. He was a senior engineer with the California NanoSystems Institue, University of California, Los Angeles, CA, USA, from 2017 to 2018. He was a Postdoctoral Research Fellow in the Department of Biomedical Engineering at the University of Southern California in 2018. He currently is an assistant professor of the Department of Electrical Engineering, National Chin-Yi University of Technology, Taichung City, Taiwan, where has been since 2019.

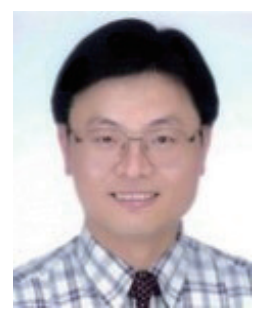

Kuei-Hsiang Chao was born in Tainan, Taiwan, in 1962. He received his B.S. degree in electrical engineering from National Taiwan Institute of Technology, Taipei, Taiwan, in 1988, and his M.S. and Ph.D. degrees in electrical engineering from National Tsing Hua University, Hsinchu, Taiwan, in 1990 and 2000, respectively. He is presently a professor at the National Chin-Yi University of Technology, Taichung, Taiwan. His areas of interest are computer-based control systems, applications of control theory, renewable energy, and power electronics. He is a life member of the Solar Energy and New Energy Association and a member of the IEEE. 


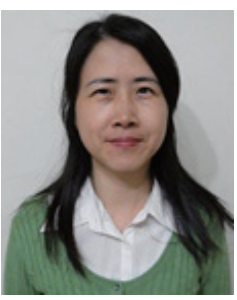

Hsiang-Yueh Lai received her Ph.D. degree from the Department of Engineering and System Science, National Tsing Hua University, Hsinchu, Taiwan, in 2010. She is presently an associate professor in the Department of Electrical Engineering, National Chin-Yi University of Technology, Taichung, Taiwan. Her current research interests include intelligent systems, embedded systems, and solid-state electronic devices.

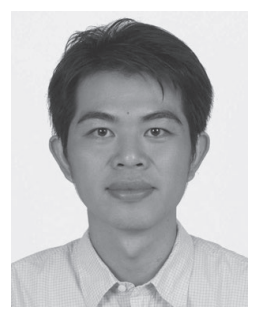

Jian-Hung Liu received his Ph.D. degree in electrical engineering in 2008 from National Taiwan University, Taipei, Taiwan. He is the founder of Asensor Corporation (Taipei, Taiwan) and was its CEO from 2009 to 2014. Asensor Corporation primarily designs and manufactures customized ultrasound transducers, photoacoustic probes, and HIFUs. He was at Qisda Corporation, Taoyuan, Taiwan, from 2014 to 2018. He established a diagnostic ultrasound transducer factory and mass-produced more than 10 models of commercial transducer. He is currently a consultant in the Electrical Engineering department of National Chin-Yi University of Technology, Taichung, Taiwan. His research interests include ultrasound transducers, acoustic output, and ultrasound images.

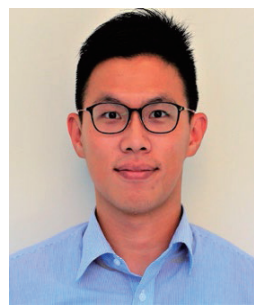

Howard Chung was born in 1987. He received his M.D. degree from Taipei Medical University in 2015, and passed the national medicine board exam in the same year and the ECFMG certification exam in 2017. He was a research fellow in the Department of Molecular and Medical Pharmacology, University of California Los Angeles, CA, from 2017 to 2018. Currently, he is affiliated with the Internal Medicine Department in Queen Hospital Center, NY, USA.

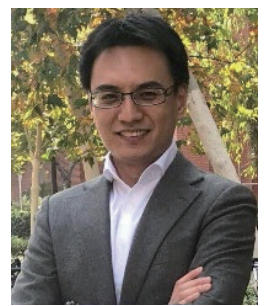

Hsiao-Chuan Liu was born in 1980. He received the M.S. degree in biomedical engineering from National Yang Ming University, Taipei, Taiwan, in 2005, and the Ph.D. degree in biomedical engineering from the University of Southern California, Los Angeles, CA, USA, in 2019. He is a Research Fellow in Mayo Clinic, Rochester, MN, USA. His current research interests include cell mechanics, acoustic tweezers, shear waver and surface wave elastography, imaging processing and clinical ultrasound. 